\title{
MENTAL COACHING
}

\section{Mentales Sport Coaching}

\section{EXERCISE IS MEDICINE}

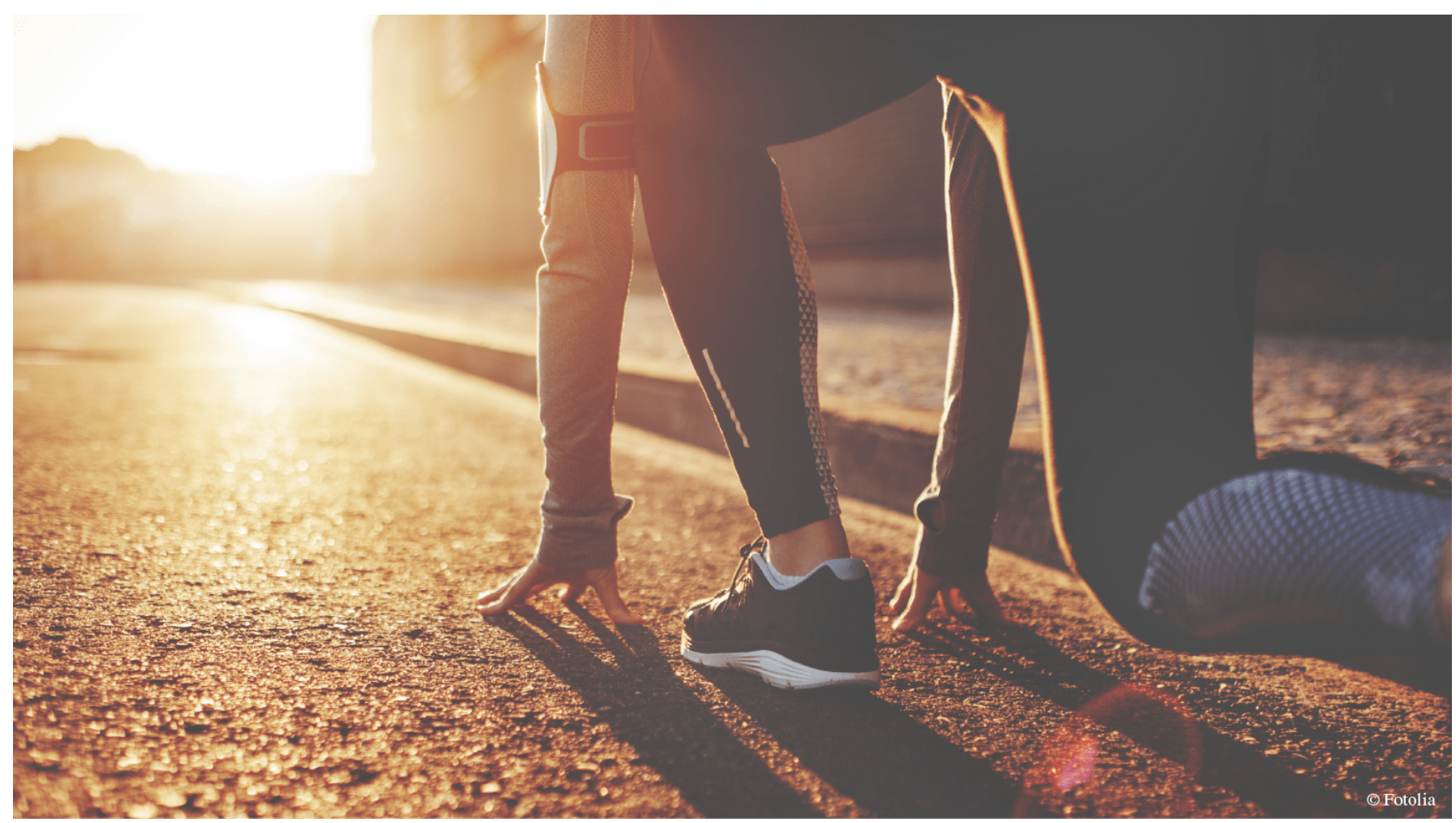

\section{Suter Monika}

Ärztezentrum Limmatfeld, Physiotherapie Limmatfeld, Dietikon [1,2]

\section{Abstract}

As a Sports Mental Coach, I would like to support athletes in their performance, in change processes as well as competently accompany them to their way and goals.

Coachings at the Limmatfeld Physiotherapy Centre, will include Thematic areas for methodo1ogical implementation and at the same time the personal development process.

Mental Training in sports is about process accompaniment to success. I accompany athletes in both grassroots and competitive sports. People in the economic environment can also benefit from sports psychology interventions in a sustainable way. [3]

"lt's won in the head, but lost as well."

Mental Strength is needed to achieve its goals. The power source sits in each one of us. In mental sports coaching,

I show how the potential of this source is being exploited and can be used, with a variety of interventions 
to achieve goals. I design an individual coachings so that the athletes can mobilize potentials in challenging situations and act in an effective way.

\section{Zusammenfassung}

Als Sport Mental Coach möchte ich Sportler in ihrer Leistungserbringung, bei Veränderungsprozessen sowie auf dem Weg zu ihren Zielen kompetent begleiten. Meine Coachings im Physiotherapiezentrum Limmatfeld sollen Themenbereiche zur methodischen Umsetzung und gleichzeitig den persönlichen Entwicklungsprozess beinhalten. Beim Mentalen Training im Sport geht es um Prozessbegleitungen zum Erfolg. Ich begleite Sportler im Breiten- wie auch im Leistungssport. Auch Menschen in der Leistungserbringung im wirtschaftlichen Umfeld können mit sportpsychologischen Interventionen nachhaltig unterstützt werden. [3]

"Gewonnen wird im Kopf, verloren aber auch.»

Mentale Stärke ist gefragt, um seine Ziele zu erreichen. Die Kraftquelle sitzt in jedem. Im Mentalen Sport Coaching möchte ich aufzeigen, wie das Potenzial dieser Quelle ausgeschöpft werden kann. Mit vielfältigen Interventionen, um Ziele umzusetzen. Ich gestalte ein individuelles Coaching, damit der Sportler in herausfordernden Situationen Potenziale mobilisieren und handlungsfähig agieren kann.

«Behandle die Menschen so, als wären sie, was sie sein sollten, und du hilfst ihnen zu werden, was sie sein könnten.»

Johann Wolfgang von Goethe

\section{Introduction}

Das mentale Training basiert auf Grundlagen der Psychologie und befasst sich mit dem menschlichen Denken und Verhalten bezüglich seiner Handlung.

Dazu werden in einem Prozess Methoden und Techniken erarbeitet, mit deren Unterstützung sich Sportler zielgerichtet vorbereiten können. Mentales Techniktraining beruht auf einer Visualisierungsmethode, mit welcher Bewegungsabläufe erlernt, stabilisiert, verändert oder perfektioniert werden.

Mentales Training muss genauso erlernt und praktiziert werden wie etwa ein Athletik- oder Techniktraining. Es stellt eine Trainingsmassnahme dar, ist aber kein Allheil- oder Wundermittel mit garantierter Sofortwirkung. Vielmehr soll es sich neben den anderen Trainingsmassnahmen als Grundbaustein etablieren.

\section{Methods}

Die mentale Betreuung gliedert sich nach Elbe und Beckmann [4] in vier Bereiche: Das Grundlagentraining, das Fertigkeitstraining, die Krisenintervention und die Diagnostik. Zum Grundlagentraining zählen hauptsächlich Entspannungsverfahren. Dazu gehören Atemtechniken, Progressive Muskelrelaxation oder autogenes Training. Diese bilden das Fundament für viele weitere Techniken aus dem Fertigkeitstraining und tragen durch ihre Stress abbauende Wirkung zu einer gesunden und ausgeglichenen Haltung bei.

Erfolgreiche Menschen wissen immer, «warum» sie genau das machen. Sie haben eine Vision von sich. 
Zeit, Tempo und der Zeitpunkt sind zentral, nicht nur im Sport. Ein Ziel zu erreichen, ist der grösste Antrieb, die stärkste Motivation die es gibt, dieses Ziel lässt erfolgreiche Menschen auch in schwierigen Phasen nicht das Handtuch werfen. Die Sache tun, der Sache willen und nicht des Gewinnens willen. Als Fertigkeitstraining versteht man das Vermitteln und Trainieren von mentalen Fertigkeiten, die für den Sport und die Leistungserbringung förderlich sind. Hierzu zählen beispielsweise das mentale Techniktraining, Psychoregulation, Konzentration, Motivation oder teambildende Massnahmen. Diese Fertigkeiten werden individuell in Coachings erarbeitet und an die jeweilige Sportart oder Problematik angepasst.

Jeder Sportler ist einzigartig und die wichtigste Person zum Erreichen seines Ziels. "Gewinnen tut man selbst, verlieren ebenso.» Verantwortung für sich selber übernehmen, was bedeutet, dass jeder für sein Scheitern selbst verantwortlich ist und nicht andere Umstände dafür herangezogen werden sollen. Dieses Denken bedeutet, dass jeder selbständig und handlungsfähig zu seinem Erfolg beitragen und diesen steuern kann.

Die Krisenintervention findet ausschliesslich in Einzelcoachings statt. Oftmals sind die Anliegen Problematiken im Umfeld des Athleten, Verletzungspausen, Ängste, Blockaden bei der Leistungserbringung oder Motivationsschwierigkeiten.

Flexible Sportler wissen, dass viele Wege nach Rom führen. Es einen oder mehrere Wege gibt, die sie beschreiten können. Ein flexibles Verhalten hilft dabei, zwischen diesen Optionen hin und her zu springen. Zuerst den einen Weg zu versuchen und falls dieser nicht erfolgsvorsprechend ist, einen anderen Weg einzuschlagen und mit diesem erfolgreich zu sein. Flexibel sein bedeutet, ausprobieren, optimieren und neugierig sein auf Chancen, die sich auf dem Weg zu Erfolg abzeichnen. Auch Sportler stecken manchmal in einer Krise, benötigen Inputs zur Veränderung ihrer Verhaltensmuster. Gerade in der Teambetreuung ist es essenziell, auch auf einzelne Sportler einzugehen und Krisen zu erkennen. Die sogenannten «red flag»-Verhaltensmuster können in drei Kategorien eingeteilt werden:

- Physische Funktion: haben die Sportler genug Regeneration, Schlaf? Stimmt ihre Energiebilanz bezüglich der Belastung, Verletzungsmanagement? Blockaden bei Wettkämpfen?

- Emotionale Funktion: reagiert der Sportler Situationsangepasst? Ist er schneller frustriert? Wie geht er mit Emotionen um? Unstimmigkeiten im sozialen Umfeld?

- Kognitive Funktion: kann er die Dinge im Team adäquat interpretieren?

Zur Krisenintervention gehören im Rahmen der Sportpsychologie oftmals Problematiken, die den Athleten in der erfolgreichen Ausführung des Sports beeinträchtigen, wie die erwähnten «red flags». Natürlich ist die Krisenintervention ein Teil der mentalen Betreuung, jedoch geht es dabei nicht um Störungen. Diese sollten nur von ausgebildeten Psychotherapeuten behandelt werden. Gegebenenfalls vermittle ich Sportler an entsprechende Therapeuten weiter.

Diagnostik kann zu verschiedenen Zwecken angewandt werden. Zum einen als begleitende Massnahme (Monitoring) zur Feststellung der Wirksamkeit der mentalen Betreuung. Zum anderen dient sie der Erfassung von IST-Werten des Athleten. Darauf aufbauend, können Ressourcen entdeckt und Potenziale erkannt werden, welche dann systematisch zur Leistungssteigerung oder -stabilisierung eingesetzt werden können.

Weitergehend beschäftigt sich das mentale Training nicht nur mit den Athleten. Auch Trainer, 
Funktionäre und Eltern sind Zielgruppen des Coachings, denn auch sie können von mentalem Training profitieren. Hierbei geht es meist um die Erarbeitung hilfreicher Strukturen, die optimale Betreuung der Sportler, erfolgreiches und authentisches Coaching sowie die eigene Präsentation nach aussen (beispielsweise im Umgang mit den Medien).

Mentales Training ist ein wichtiger Bestandteil des modernen Sports, genauso wie Athletik- und Taktiktraining, oder Ernährungsberatung und Physiotherapie im Aerztezentrum Limmatfeld. Es ist ein Baustein von vielen, der einen grossen Beitrag dazu leistet, dass ein Sportler zum richtigen Zeitpunkt seine Spitzenleistungen abrufen kann.

\section{Results}

Mentale Interventionen dienen der Optimierung mentaler Leistungsvoraussetzung und der Erreichung momentaner Maximalleistung. Der Zielsetzung, dem Optimieren und Neuerwerben von Bewegungsabläufen, dem Training mentaler Fähigkeiten, dem Aufrechterhalten und Wiederherstellen eines gesunden mentalen Zustandes sowie der Unterstützung bei der Persönlichkeitsentwicklung.

\section{Discussion}

Was bringt die Begleitung durch einen Sport Mental Coach? Mein Begleitungsverständnis: Menschen handeln, fühlen und denken grundsätzlich so, wie es ihrer gelernten Einstellung entspricht. Ich gehe immer mit der Annahme auf den Sportler zu, dass er die Lösung selbst in sich trägt. Wenn er zu mir kommt, braucht er keine Probleme, sondern den Willen, sich weiterentwickeln zu wollen. Was macht der mentale Faktor im Bezug auf Leistung aus? Ist Erfolg eine Frage des Talents, der Einstellung oder einfach nur des Glücks? Es ist nicht nur eine Frage des Glücks. Das mind set und die physische Konstellation sind die zwei Seiten einer Medaille und untrennbar miteinander verbunden. Während einer Performance körperlich und mental eine Einheit zu sein und dadurch Bestleistung, auch zum Zeitpunkt X abrufen zu können - Das ist Erfolg und für jeden lernbar. Und genau da beginnt meine Motivation und Arbeit als Sport Mental Coach.

Durch ihre Qualifikation konnte eine meiner Coaches mit der Schweizer Delegation von Swiss Olympic an das Europäische Olympische Jugendfestival, EYOF 2019 in Baku. Mit dieser Athletin (Swiss Starter Future, Nationalkader Mehrkampf, Nationalstaffel 4 x 100 m) habe ich als mentale Vorbereitung, bezüglich optimalem Leistungszustand gearbeitet. Sehr spezifisch auf eine ihrer Disziplinen innerhalb des 7-Kampfs. Ein Ziel des mentalen Trainings ist es, dass die Sportler ihre im Moment vorhandenen Kompetenzen punktgenau auf einen fremdbestimmten Zeitpunkt entfalten können, der sogenannte OLZ (optimale Leistungszustand). Die Wettkampfresultate mit Saisonbestleistung waren als Feedback für die Athletin und für meine Arbeit als Coach sehr befriedigend und zeigen die Möglichkeiten des Mentalen Trainings im Sport sehr gut auf. [5]

\section{Acknowledgments, Vorgehensweise Coaching}

Phase 1: Kontaktaufnahme und Erstgespräch

Das kostenlose Erstgespräch besteht aus einem persönlichen Termin, an dem ich die Sportler gerne 
kennenlerne - sowie sie mich. Mentales Training ist Vertrauenssache. Dass es zwischen uns passt, ist für eine erfolgreiche Zusammenarbeit unabdingbar.

\section{Phase 2: Analyse der persönlichen Situation}

Das eigentliche Coaching beginnt mit einer ausführlichen Analyse/Anamnese der Ist-Situation. Wo steht der Sportler zurzeit? Worin liegt sein Fokus? Wo möchte er hin? Was möchte er erreichen? Anhand dieser Analyse wird gemeinsam ein Endziel und möglicherweise werden auch Etappenziele vom Athleten definiert. Diese Auftragsklärung dient als Grundlage und ist die Basis für ein erfolgreiches Mentaltraining.

\section{Phase 3: Mit zielführenden Massnahmen zum Erfolg}

In der dritten Phase geht es darum, den Sportler mit zielgerichteten Interventionen in seinem mentalen Prozess voranzutreiben. Mental Coachings und Support beim Aufbau einer positiven Trainings- und Leistungsstruktur. Wir besprechen, planen und entscheiden gemeinsam. Wir setzen um, kontrollieren und nehmen bei Bedarf Anpassungen vor. So kann sichergestellt werden, dass der Prozess in die richtige Richtung läuft. Mental stark zu werden ist ein Prozess, welcher Geduld, Wille und Mitarbeit erfordert.

\section{Corresponding author}

Monika Suter

Dipl. Sport Mental Coach, Dipl. Coach SCA, Betriebliche Mentorin eidg. FA Fuchsweidstrasse 18, 8967 Widen Tel. 0763841465

E-Mail: suter@mentalsportcoach.ch $\mathrm{B}$

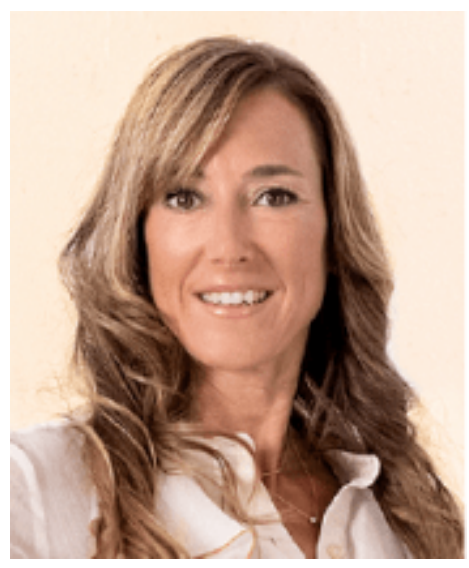

\section{References}

1. http://www.aerztezentrum-limmatfeld.ch

2. http://www.aerztezentrum-limmatfeld.ch/Angebot/Physiotherapie/index.html

3. https://mentalsportcoach.ch

4. https://www.netzathleten.de/fitness/richtig-trainieren/item/3905-angewandte-sportpsychologie-was-ist-das-wirklich. Literaturangabe: Beckmann, J., Elbe, A. (2011). Praxis der Sportpsychologie. Mentales Training im Wettkampf- und Leistungssport. Balingen: Spitta Verlag (2. erw. Auflage).

5. https://www.swissolympic.ch/ueber-swiss-olympic/news-medien/medienmitteilungen/ 2019/EYOFBaku-2019-Gute-Erinnerungen-und-neue-Erfahrungen 
ATHLETE SUPPORT COMPETITION PREPARATION MENTAL COACHING PERSONAL DEVELOPMENT 